\title{
THE EFFECT OF REGIONAL ANAESTHESIA AND GENETIC FACTORS ON THE DEVELOPMENT OF CHRONIC PAIN FOLLOWING TOTAL KNEE ARTHROPLASTY
}

\author{
S.C. Sciberras ${ }^{1}$, B. Vella ${ }^{2}$, A.P. Vella², J.A. Spiteri², C. Mizzi², K. Borg Xuereb², G. Laferla1', G. Grech ${ }^{3}$ \\ ${ }^{1}$ Department of Surgery, Faculty of Medicine and Surgery- University of Malta, Msida, Malta \\ ${ }^{2}$ Department of Anaesthesia, Intensive Care and Pain Management, Mater Dei Hospital, Msida, Malta \\ ${ }^{3}$ Department of Pathology, Faculty of Medicine and Surgery- University of Malta, Msida, Malta
}

\section{BACKGROUND AND AIMS}

Multiple factors, both peri-operative ${ }^{1}$ and genetic ${ }^{2}$, have been attributed to the development of chronic pain after surgery. This research investigates the influence of anaesthesia and genetic factors on chronic postsurgical pain (CPSP) after Total knee arthroplasty (TKA).

\section{METHODS}

In this randomised controlled study, 199 patients undergoing elective TKA were randomized to receive either spinal anaesthetic (Group SP, $n=109$ ) or a general anaesthetic with femoral block (Group GA, n=90) (Figure 1). Baseline demographics included age, surgical firm, gender, BMI, ASA, WOMAC ${ }^{3} 3$ and S-LANSS. A standard postoperative analgesic plan was used for all patients. Patients were followed up for 24 hours, and again at three and at six months by a telephone interview, assessing WOMAC® and S-LANSS

Genotyping was performed using real-time PCR using TaqMan ${ }^{\circledR}$ probes for various single nucleotide polymorphisms (SNP) in the COMT and SCN9A genes

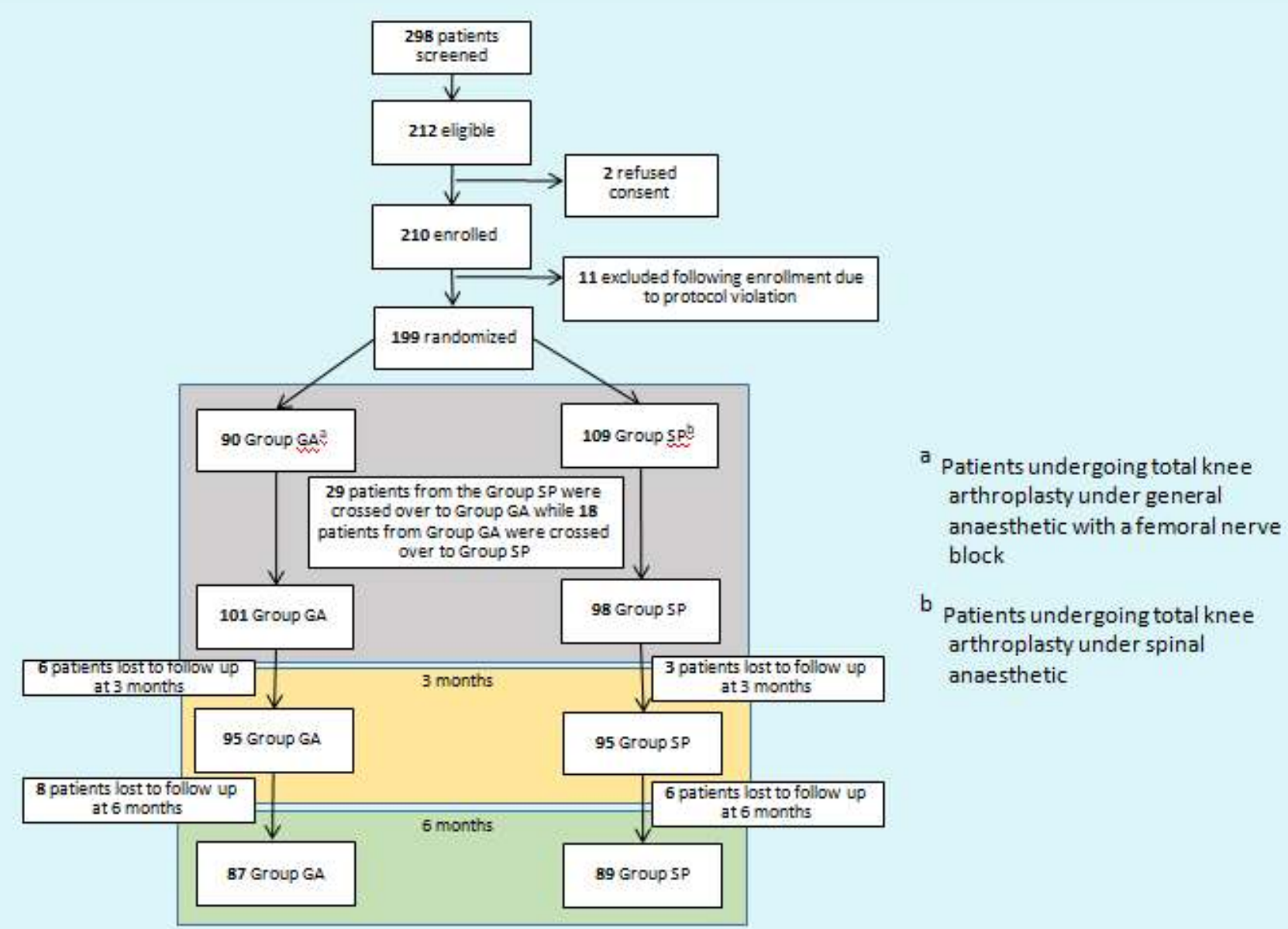

Figure 1 - CONSORT diagram showing enrolment of subjects and analysis during the trial

\section{RESULTS}

The demographics for the study group was similar to those reported in other centres with a mean age of 66 years, a female to male ratio of $2: 1$, a predominant ASA of I-II and a WOMAC score of 42 consistent with recommendations of when to perform surgery.

Primary outcome was the difference from baseline of the WOMAC $®$ Pain sub score after three and six months. We also investigated the incidence of CPSP at six months, defined as WOMAC $®$ Pain score greater than 5
Anaesthesia and chronic pain

Patients undergoing spinal anaesthesia had a larger decrease in the WOMAC $\circledR$ score at both intervals, but this was statistically significant only at three months. (Three months: 7.4 vs $6,95 \% \mathrm{Cl}=-0.06$ to $2.5, \mathrm{p}<0.001$; Six months: 8 vs $7,95 \% \mathrm{Cl}=-0.2$ to $2.1, \mathrm{p}=0.09$ ) as seen in Figure 2.

The incidence of CPSP at six months was also lower in Group SP on multivariate analysis (OR $0.34,95 \% \mathrm{Cl}=0.11$ to $0.96, p=0.048)$. The main three factors that could predict the incidence of chronic post surgical pain at six months were baseline pain, with an increase in OR of $35 \%$ for every unit on WOMAC® pain sub score, pain at three months after surgery, with $77 \%$ increase in likelihood for every unit of pain and type of anaesthesia, with a spinal anaesthetic having a $90 \%$ less risk.

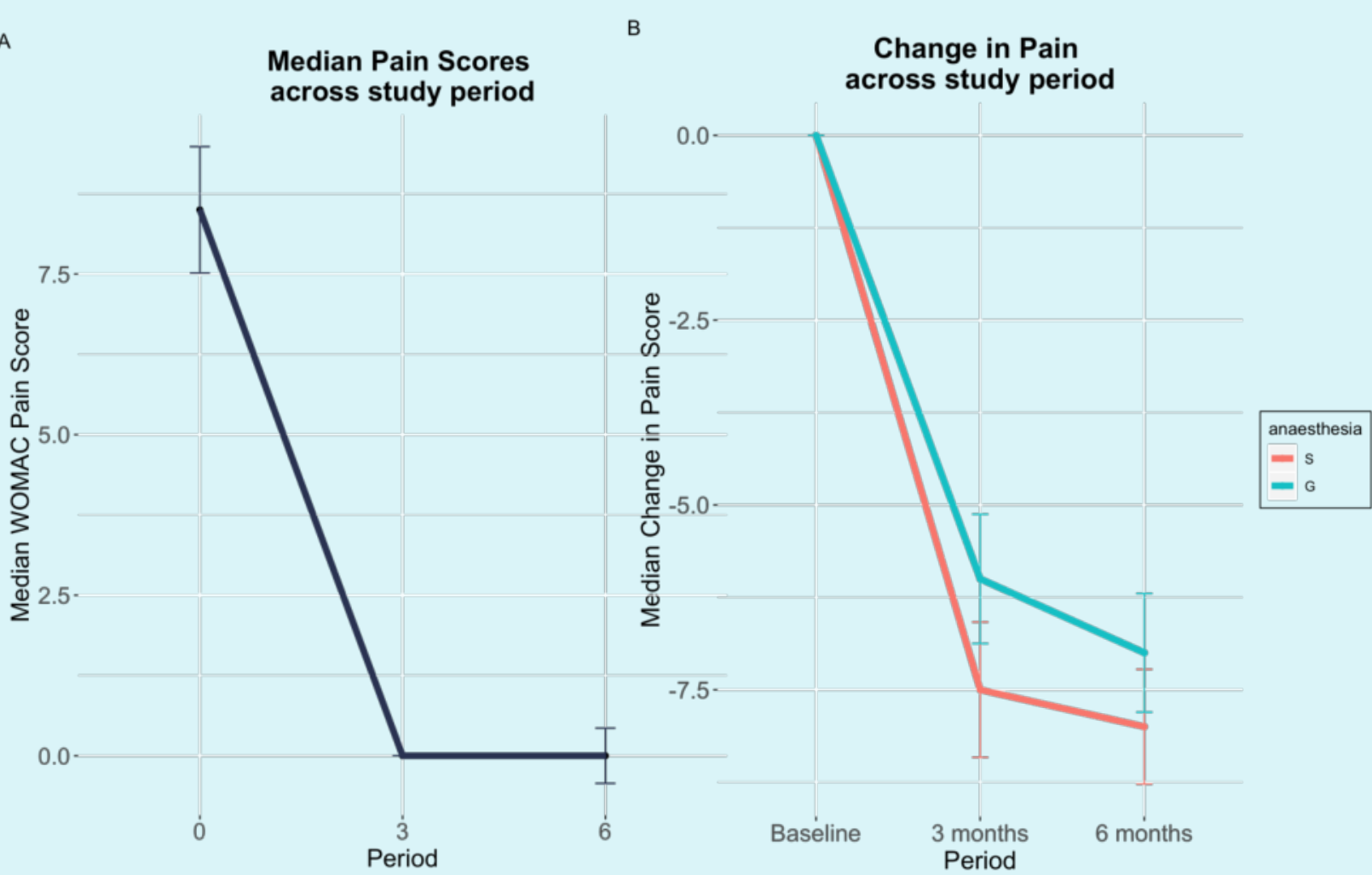

Figure 2 - A: median WOMAC pain scores and $B$ : Change in median WOMAC pain scores at 3 and 6 months

\section{Genetic influence and chronic pain}

Carriers of the rs11898284 variant of the SCN9A gene had increased pain scores at rest at three months $(36 \%$ vs $16 \%, p=0.003)$ and six months $(32 \%$ carriers vs $13 \%$ non-carriers, $p=0.01$ ).

Carriers of the rs 4818 variant of the COMT gene had increased WOMAC ${ }^{8}$ pain scores at 6 months (2 vs 1 , $\mathrm{p}=0.0028)$

\section{CONCLUSION}

Spinal anaesthesia might be beneficial in reducing the onset of CPSP, as defined by a pain subset of WOMAC $尺$ greater than 5 . Genetic factors seem to play a role in patient outcomes following total knee arthroplasty

This research may help identify patients at risk of CPSP and individualize peri-operative care and followup in such patients 